Advance Journal of Food Science and Technology 5(2): 84-89, 2013

DOI:10.19026/ajfst.5.3224

ISSN: 2042-4868; e-ISSN: 2042-4876

(C) 2013 Maxwell Scientific Publication Corp.

Submitted: June 28, $2012 \quad$ Accepted: July 28, $2012 \quad$ Published: February 15, 2013

Research Article

\title{
Effects of Salt on Wheat Flour Dough Fermentation
}

\author{
Toshiyuki Toyosaki and Yasuhide Sakane \\ Department of Foods and Nutrition, Koran Women's Junior College, Fukuoka 811-1311, Japan
}

\begin{abstract}
Most food chemistry characteristics in the dough fermentation of salt are not solved. Effects of salt on the acceleration process of wheat flour dough fermentation were studied, respectively. The mechanism of dough expansion influenced by salt and yeast was also investigated. The dough expansion rate with no salt reached a maximum of $18 \%$ in the 50 min dough fermentation time. In contrast, dough with $2.0 \%$ salt reached an expansion rate of $96 \%$ in 30 min of fermentation. Furthermore, the maximum dough expansion rate with $8.0 \%$ salt was $58 \%$ in $20 \mathrm{~min}$. Lipid peroxidation catalyzed by baker's yeast was observed in the dough fermentation process following the addition of salt. Although the baker's yeast catalyzed lipid peroxidation salt triggered the reaction. The hydroperoxide produced in the induced lipid peroxidation reaction was found to play an unspecified role in the expansion phenomenon of dough. Based on these findings, we examined how salt is associated with the dough fermentation phenomenon. We hypothesized that the presence of salt would induce the following two chemical phenomena: 1) Salt enhances cross-linkages between gliadin and glutelin, which in turn leads to increased gluten content. 2) While baker's yeast catalyzes lipid peroxidation, salt potentiates this reaction. We speculated that hydroperoxide, produced in lipid peroxidation, would accelerate the dough fermentation process, thereby resulting in a higher dough expansion rate. These results revealed some new findings in the biochemical effects of salt in bread making, which could break new ground in the bread-making industry.
\end{abstract}

Keywords: Expansion, fermentation, hydroperoxide, lipid peroxidation, salt, wheat dough

\section{INTRODUCTION}

In the field of food science, salt is generally considered to be an important chemical compound (additive), since it not only affects palatability or taste, but also affects the structural and physical properties of food. There is a long history of excellent studies documenting the various properties of salt (Cardoso et al., 2008; Mercier and Gelinas, 2001; Gou et al., 2003; Ishikawa et al., 2006; Kuda et al., 2007; Murphy et al., 1998; Rowe et al., 2009; Shigematsu et al., 2007; Sofos, 1983; Steel and Torrie, 1980; Kaewmanee et al., 2011; Toyosaki et al., 2006; Ukai et al., 2008; Vardhanabhuti and Foegeding, 2008; Wang et al., 2011). In our study, a number of interesting findings have suggested that the presence of salt in the fermentation process accelerates dough fermentation. This phenomenon suggests a new biochemical effect of salt. In general, salt has commonly been used as an essential secondary ingredient in bread making. However, little is known about the biochemical effect of salt in the bread making process. As a result of investigating the function of added salt in bread making, we unraveled the critical role of salt in dough fermentation. However, the mechanism of action for this phenomenon and the relationship between salt and the flavor and texture of baked breads remain unknown. A thorough investigation of these issues from various angles led to some interesting findings.

\section{MATERIALS AND METHODS}

Materials: Spring wheat flour (Super King; 13.8\% protein, $0.42 \%$ ash, $14 \%$ water) was obtained from Nisshin Flour Milling Inc. (Chiyoda, Tokyo, Japan). Dried yeast (Saccharomyces cerevisiase) was purchased from S.I. Lesaffre (Marcq-en-Baroeul, France). Lipoxygenase (from soybean, Type I-B, lyophilized) and linoleic acid (99\% pure) were obtained from Sigma Chemical Co. (St. Louis, MO, U.S.A). Gluten (more than $90 \%$ pure), 2', 7'-Dichlorofluorescein (DCF) and hematin (more than $90 \%$ pure) were purchased from Nakarai Tesque, Inc. (Kyoto, Japan). Silicagel 70 F254 $(20 \times 20 \mathrm{~cm})$ ware purchased from Wako Pure Chemical Industries, Ltd. (Tokyo, Japan). Other reagents were of special grade and were obtained from Nacalai Tesque, Inc. (Kyoto, Japan).

Preparation of dough: The wheat flour that was used to make adjustments in the bread dough was the type that is readily commercially available. The salt was added at 2.0 and $8.0 \%$ level. The fats added was butter,

\footnotetext{
Corresponding Author: Toshiyuki Toyosaki, Department of Foods and Nutrition, Koran Women's Junior College, Fukuoka 811-1311, Japan

This work is licensed under a Creative Commons Attribution 4.0 International License (URL: http://creativecommons.org/licenses/by/4.0/).
} 
which was added at a 3.0-8.0\% level. Other ingredients used to make the bread were all commercially available. The yeast content was added at the $0-10 \%$ of bread dough adjustment and underwent primary fermentation in an incubator at $37^{\circ} \mathrm{C}$ with $75-80 \%$ humidity. After fermentation, gas was released; after a bench time of $10 \mathrm{~min}$, the dough underwent final fermentation for $50 \mathrm{~min}$ and was then baked for $12 \mathrm{~min}$ at $200^{\circ} \mathrm{C}$.

Preparation of linoleic acid hydroperoxide: Linoleic acid hydroperoxide was prepared according to the produce of Matsuda et al. (1978) by enzymic per oxidation with lipoxygenase. The standard reaction mixture, containing $32 \mathrm{mM}$ linoleic acid ( $99 \%$ pure), $0 / 1 \%$ Tween $80,0.2 \mathrm{M}$ borate buffer $(\mathrm{pH} \mathrm{9.0)}$ and 50 units of lipoxygenase in a total volume $20 \mathrm{~mL}$, was incubated in a conical flask to facilitate its being flushed with pure oxygen. The reaction mixture was stirred mechanically for $40 \mathrm{~min}$ at $30^{\circ} \mathrm{C}$ under a stream of pure oxygen in a separating funnel. Then hydroperoxide was extracted with diethyl ether and concentrated by removal of the solvent. The formation of linoleic acid hydroperoxide was monitored by measurement of the increase in the absorbance at 234 $\mathrm{nm}$. Absorbance at $234 \mathrm{~nm}$ was used as direct index of the amount of the conjugated diene and as an indirect index of the amount of hydroperoxide. The hydroperoxide was checked by TLC for purity with a mixture of $n$-hexane diethyl ether and acetic acid $(60: 40: 1, \quad \mathrm{v} / \mathrm{v} / \mathrm{v})$ as the solvent system and was monitored under UV light.

Preparation of the model system of linoleic acid hydroperoxide: By mixing a fixed amount of commercially available materials and linoleic acid hydroperoxide of $0-100 \mathrm{mM}$ level, which served as the test sample, a model system was created.

Measurement of the rate of dough expansion: To determine the rate of dough expansion with fermentation, a fixed amount of dough was placed in a graduated cylinder and fermented in an incubator (temperature $30^{\circ} \mathrm{C}$, humidity $75 \%$ ). The rate of expansion over a fixed period of time was then measured.

Measurement of hydroperoxide: Hydroperoxide concentration was calculated in terms of 2', 7'Dichlorofluorescein (DCF). To de-emulsify, the $5.0 \mathrm{~mL}$ samples were centrifuged $(10,000 \times \mathrm{g}, 30 \mathrm{~min})$. The linoleic acid of the supernatant was then measured to determine the hydroperoxide level using the method of Cathcart et al. (1984). First, $1.0 \mathrm{~mL}$ of a $1.0 \mathrm{mM}$ solution of DCF in ethanol and $2.0 \mathrm{~mL}$ of $0.01 \mathrm{~N} \mathrm{NaOH}$ were mixed and stirred for $30 \mathrm{~min}$ before being neutralized with $10 \mathrm{~mL}$ of $25 \mathrm{mM}$ phosphate buffer $(\mathrm{pH}$ 7.2). Then $2.0 \mathrm{~mL}$ of the neutralized DCF solution were added to a solution of hematin $(10 \mathrm{mM})$ in $25 \mathrm{mM}$ phosphate buffer (pH 7.2; $0.01 \mathrm{mg} \quad \mathrm{DCF} / \mathrm{mL}$ ); subsequently, $2.0 \mathrm{~mL}$ of this hematin-DCF solution and $10 \mathrm{~mL}$ of the linoleic acid sample were mixed and left at $50^{\circ} \mathrm{C}$ for $50 \mathrm{~min}$, before fluorometry treatment (excitation: $400 \mathrm{~nm}$; emission: $470 \mathrm{~nm}$ ) to measure DCF. This method measures hydroperoxide with more sensitivity than the iron rhodanide method that is usually used.

Statistical analysis: Analysis of Variance (ANOVA) was performed and means comparisons were obtained by Duncan's multiple range test (Steel and Torrie, 1980). Significance was established at $p<0.05$.

\section{RESULTS AND DISCUSSION}

The effect of salt concentration on the dough expansion rate: To determine the effects of salt concentration on dough fermentation, dough test samples with varying salt concentrations were prepared and fermented under certain humidity and temperature conditions. The dough expansion rates were measured and the results are shown in Fig. 1. The dough expansion rate with no salt reached a maximum of $18 \%$ in the 50 min fermentation time. In contrast, the dough with $2.0 \%$ salt reached an expansion rate of $96 \%$ in 30 min. Furthermore, the maximum expansion rate of dough with $8.0 \%$ salt was $58 \%$ in $20 \mathrm{~min}$. The results indicated an optimal salt concentration of $2.0 \%$ for bread making in this study.

Induced lipid peroxidation associated with fermentation and hydroperoxide production: Lipid peroxidation catalyzed by baker's yeast has been a known phenomenon observed in the fermentation of dough with added salt. Under the same experimental conditions as in Fig. 1, the amount of hydroperoxide was measured and the results are shown in Fig. 2. In the $50 \mathrm{~min}$ fermentation time, a maximum of $11 \%$ of hydroperoxide was produced as a result of lipid peroxidation in dough with no salt. In contrast, a maximum value of $94 \mathrm{mM}$ was obtained for the dough with $2.0 \%$ salt in a $40 \mathrm{~min}$ fermentation time. In the dough with $8.0 \%$ salt, a maximum of $32 \mathrm{mM}$ of hydroperoxide was produced. The results demonstrate that hydroperoxide, which was produced as a result of lipid peroxidation catalyzed by baker's yeast, plays an unspecified role in the expansion of the dough. We further examined the fact that hydroperoxide, produced during lipid peroxidation, is somehow 


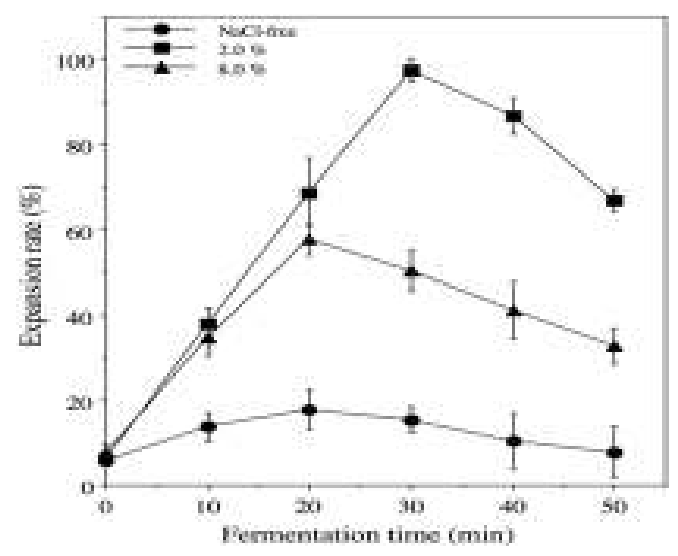

Fig. 1: Changes in the expansion rate with the fermentation of dough

Each value represents the mean \pm standard error in triplicate

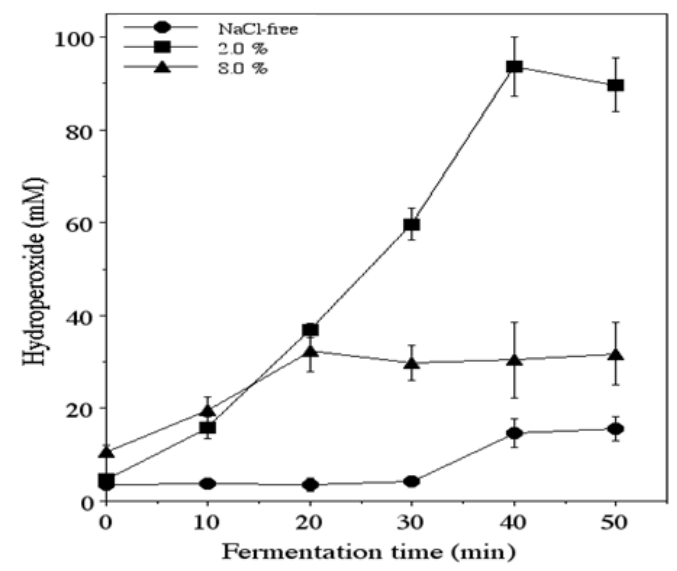

Fig. 2: Changes in the amount of hydroperoxide produced with the fermentation of dough

Each value represents the mean \pm standard error in triplicate

involved in the dough fermentation phenomenon. Using peroxide prepared from linoleic acid, the relationship between the amount of peroxide and the dough expansion rate was investigated. We observed an upward trend in the dough expansion rate with increased addition of peroxide. In particular, an expansion rate of $90 \%$ was observed in the dough with $2.0 \%$ salt with the addition of $100 \mathrm{mM}$ of peroxide (Fig. 3).

The effect of salt concentration on hydroperoxide and the expansion rate of dough: The effects of salt in dough fermentation were investigated and the results are shown in Fig. 4. The largest amount of hydroperoxide was produced from the dough with $2.0 \%$ salt, in which the highest dough expansion rate was also observed. In other words, it was indicated that the

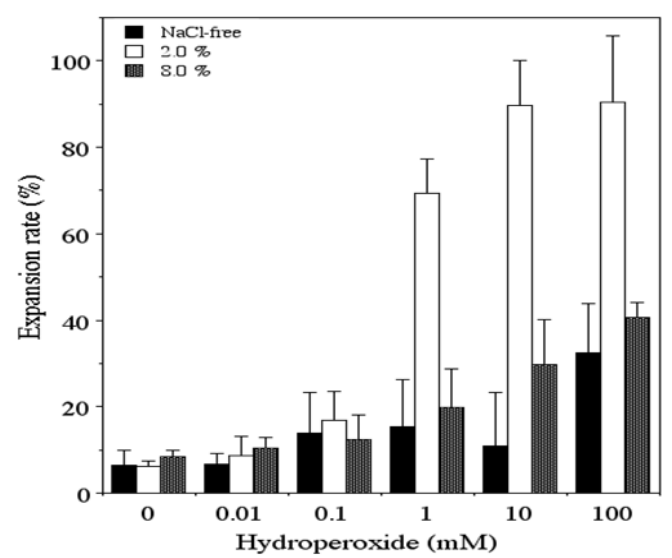

Fig. 3: Changes of expansion rate on the amount of hydroperoxide produced in dough

Each value represents the mean \pm standard error in triplicate

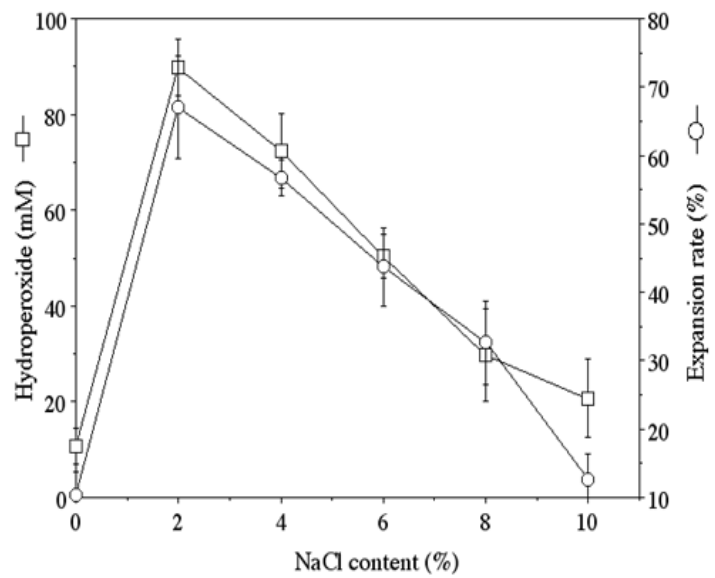

Fig. 4: Relationship between the hydroperoxide and expansion rate on the $\mathrm{NaCl}$ concentration of dough Each value represents the mean \pm standard error in triplicate

hydroperoxide produced as a result of dough fermentation induced the expansion of the dough, resulting in the increase in the dough expansion rate. We also found that this phenomenon was influenced by salt. If the hydroperoxide, produced as a result of a reaction induced by baker's yeast, was responsible for the increase in the dough expansion rate, the difference in the amount of yeast used would affect the dough expansion rate. This hypothesis was examined in detail.

Changes in the dough expansion rate depending on the quantity of baker's yeast used: The dough expansion rate with various concentrations of salt and baker's yeast (0-8.0\%) was measured (Fig. 5). The 


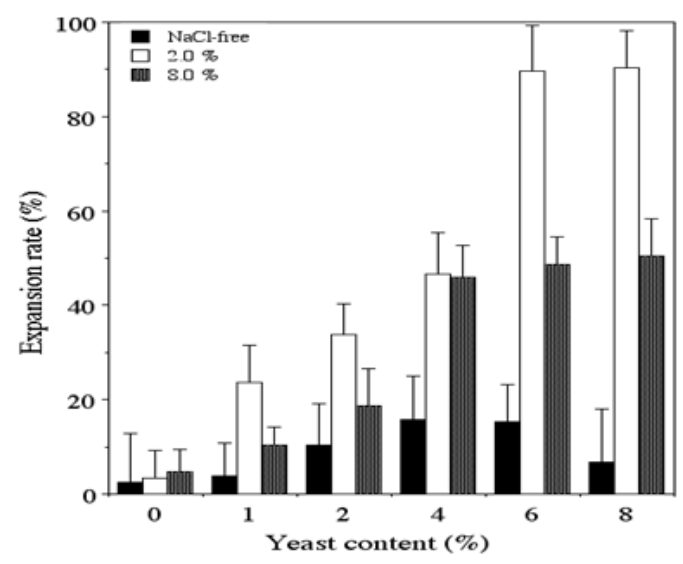

Fig. 5: Effect of yeast contents on the rate of dough expansion

Each value represents the mean \pm standard error in triplicate

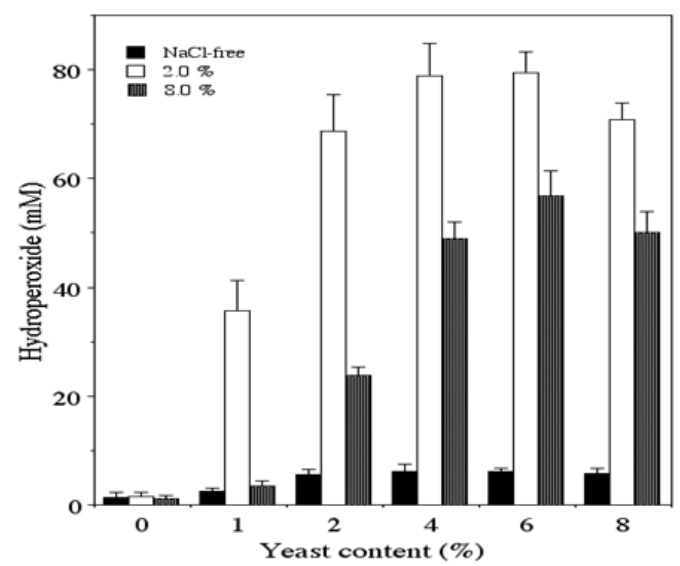

Fig. 6: Effect of yeast contents on the amount of hydroperoxide produced in dough

Each value represents the mean \pm standard error in triplicate

dough expansion rate increased as the quantity of baker's yeast increased. In the dough with $2.0 \%$ salt, in particular, an expansion rate of $90 \%$ was observed upon addition of 6.0 to $8.0 \%$ baker's yeast. The amount of hydroperoxide was measured in these experiments and the results are shown in Fig. 6. We observed an increase in hydroperoxide as dough expansion rates increased. Most notably, in the dough with $2.0 \%$ salt, $\geq 70 \mathrm{mM}$ of hydroperoxide was produced when 4 to $8 \%$ baker's yeast was used. In turn, the effect of baker's yeast on the hydroperoxide level and dough expansion rate was investigated. The amount of peroxide increased when the level of baker's yeast was between 6 and $10 \%$, which was accompanied with an increase in dough expansion rates (Fig. 7).

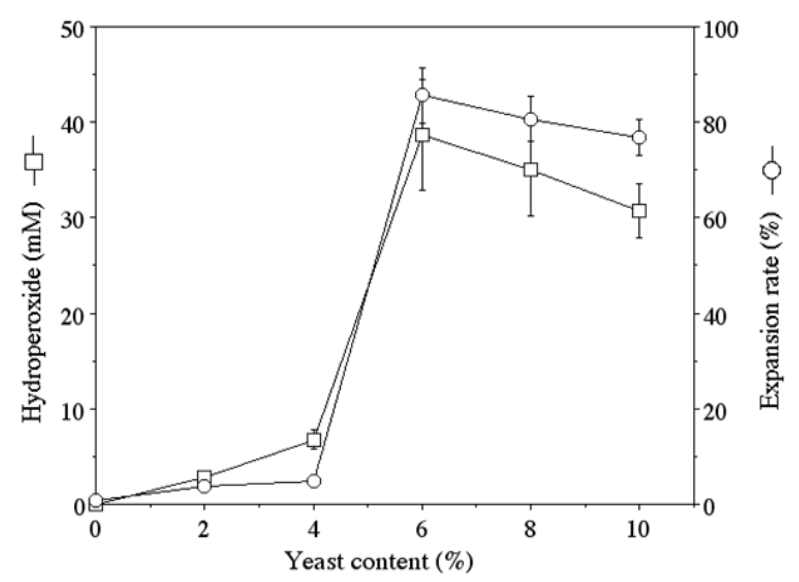

Fig. 7: Relationship between the hydroperoxide and expansion rate on the yeast contents in dough

Each value represents the mean \pm standard error in triplicate

Mechanism of dough expansion acceleration by salt: We examined how salt is associated with the dough expansion phenomenon (Fig. 8). The following events take place in the presence of salt:

- Salt enhances cross-linkages between gliadin and glutelin, which in turn leads to increased gluten content.

- Hydroperoxide is produced as a result of baker's yeast-induced lipid peroxidation. Based on the previous findings (Toyosaki and Sakane, 2002) by the author confirming the antioxidant effect of salt on lipids, slight differences in salt content may determine the activation of either antioxidative or oxidative action.

In this study, 2.0\% salt induced lipid peroxidation. However, the anti oxidative action may have inhibited the production of hydro peroxide at $8.0 \%$ salt. In any case, the expansion was accelerated in the dough with $2.0 \%$ salt by hydroperoxide produced through fermentation, which we believe led to the formation of smooth dough.

\section{CONCLUSION}

This study demonstrated a new chemical property of salt. Salt enhanced gluten-forming ability during dough fermentation. Furthermore, salt triggered lipid peroxidation catalyzed by baker's yeast, leading to the production of hydroperoxide (Fig. 8). The study demonstrated that the hydroperoxide produced in this reaction accelerated the fermentation process. Further 


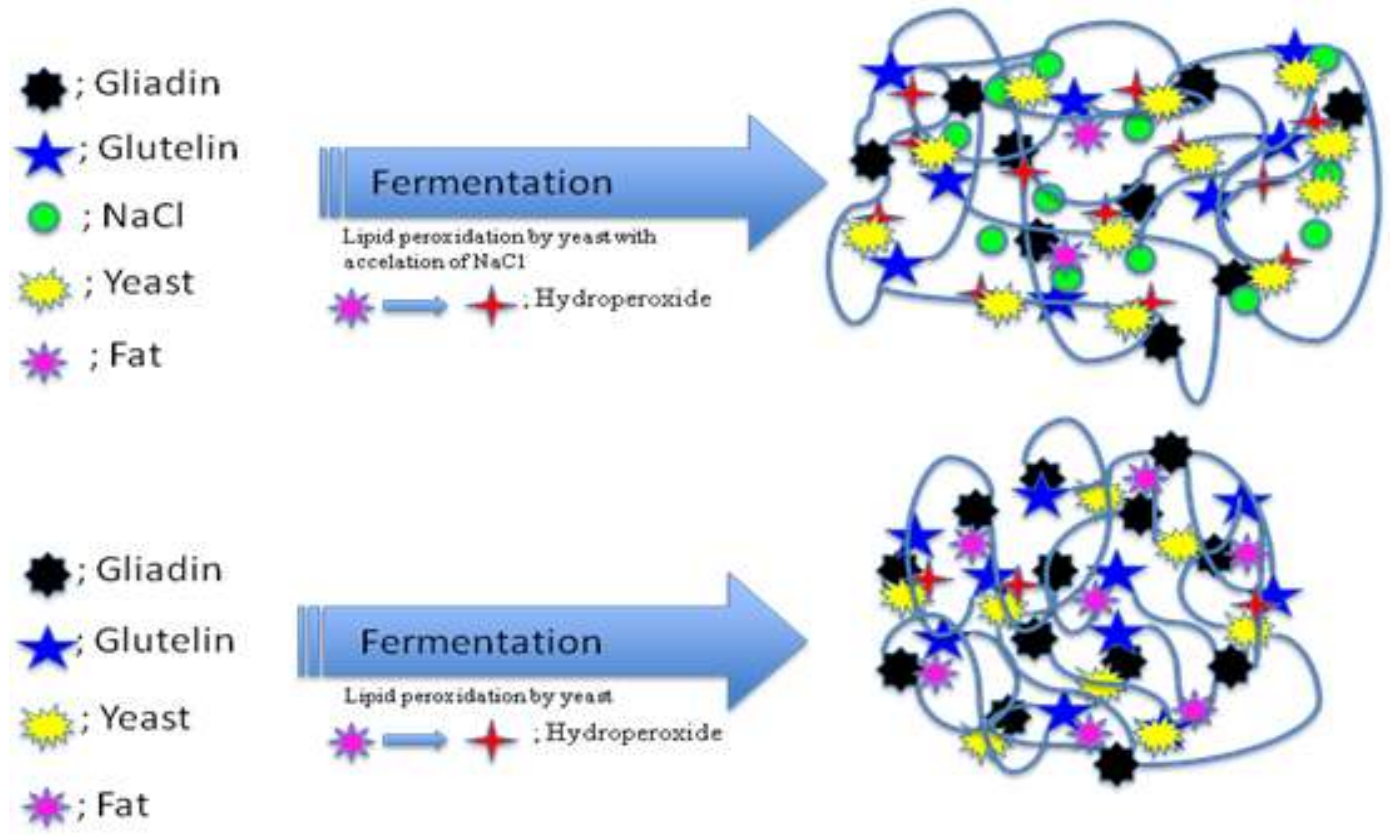

Fig. 8: The mechanism by which $\mathrm{NaCl}$ accelerate fermentation

studies are needed to investigate how salt molecules are associated with gluten formation during dough fermentation and how varying salt concentrations activate the opposing chemical actions of lipid oxidation and anti-oxidation.

\section{ACKNOWLEDGMENT}

This study was supported by a The Salt Science Research Foundation to which we are grateful.

\section{REFERENCES}

Cardoso, S.M., I. Mafra, A. Reis, D.M.R. Georget, A.C. Smith, K.W. Waidron and M.A. Coimbra, 2008. Effect of dry-salt processing on the textural properties and cell wall polysaccharides of cv: Thasos black olives. J. Sci. Food Agric., 88(12): 2079-2086.

Cathcart, R., E. Schwiers and B.N. Ames, 1984. Detection of Picomole Levels of Lipid Hydro Peroxides using a Dichloro-Fluorescein Fluorescent Assay. In: Packer, L. (Ed.), Methods in Enzymology. Academic Press Inc., New York, 105: 352-358.

Gou, P.J., J. Comaposada and J. Arnau, 2003. NaCl content and temperature effects on moisture diffusivity in the Gluteus medus muscle of pork ham. Meat. Sci., 63(1): 29-34.
Ishikawa, K., M. Sigimoto, M. Kumagai and R. Matsunaga, 2006. Evaluation of taste properties of commercially available salts. Int. J. Soc. Mater. Eng. Resour., 13(2): 29-34.

Kaewmanee, T., S. Benjakul and W. Visessanguan, 2011. Effects of salting processes and time on the chemical composition, textural properties and microstructure of cooked duck egg. J. Food Sci., 76: 139-147.

Kuda, T., T. Mihara and T. Yano, 2007. Detection of histamine and histamine-related bacteria in fishnukazuke, a salted and fermented fish with ricebran, by simple colorimetric microplate assay. Food Control, 18(6): 677-681.

Matsuda, Y., T. Beppu and K. Arima, 1978. Crystallization and positional specificity of hydro peroxidation of fusarium lipoxygenase. Biochim. Biophys. Acta, 530(3): 439-450.

Mercier, M. and P. Gelinas, 2001. Effect of lipid oxidation on dough bleaching. Cereal Chem., 78(1): 36-38.

Murphy, A., J.P. Kerry, J. Buckley and I. Gray, 1998. The antioxidative properties of rosemary oleoresin and inhibition of off-flavors in precooked roast beef slices. J. Sci. Food Agric., 77(2): 235-243.

Rowe, C.W., F.W. Pohlan, Jr. A.H. Brown, Z.B. Johnson, S.H. Whiting and D.L. Galloway, 2009. Effects of conjugated linoleic acid, salt and sodium tripolyphosphate on physical, sensory and instrumental color characteristics of beef striploins. J. Food Sci., 74(1): 36-43. 
Shigematsu, T., S. Ueno, Y. Tsuchida, M. Hayashi, H. Okonogi, H. Masaki and T. Fujii, 2007. Comparative analyses of viable bacterial counts in foods and seawater under micro plate based liquidand conventional agar plate cultivation: Increased culturability of marine bacteria under liquid cultivation. Biosci. Biotechnol. Biochem., 71(12): 3093-3097.

Sofos, J.N., 1983. Effects of reduced salt $(\mathrm{NaCl})$ levels on the stability of frankfurters. J. Food Sci., 48(6): 1684-1691.

Steel, R.G.D. and J.H. Torrie, 1980. Principles and Procedures of Statistics: A Biometrical Approach. 2nd Edn., McGraw-Hill, New York, ISBN13: 9780070609266.

Toyosaki, T. and Y. Sakane, 2002. Antioxidant effect of $\mathrm{NaCl}$ on the aqueous solution, emulsified and enzymic lipid oxidation. Bull. Soc Sea. Water Sci., 56(1): 10-16.
Toyosaki, T., Y. Sakane and M. Koketsu, 2006. Effects of addition of salt to bread on IgE antibody response. Food Agric. Immunol., 17(3): 149-156.

Ukai, T., Y. Matsumura and R. Urade, 2008. Disaggregation and reaggregation of gluten proteins by sodium chloride. J. Agric. Food Chem., 56: 1122-1130.

Vardhanabhuti, B. and E.A. Foegeding, 2008. Effects of dextran sulfate, $\mathrm{NaCl}$ and intial protein concentration on thermal stability of $\beta$ lactoglobulin and $\alpha$-lactalbumin at neutral $\mathrm{pH}$. Food Hydrocolloid., 22(5): 752-762.

Wang, Z., Y. Xu, J. Zhang, X. Li, Z. Lin and C. Ma, 2011. Proteolysis protein oxidation and protease activity in dry-cured Xuanwei ham during the salting stages. Int. J. Food. Sci. Technol., 46(7): 1370-1377. 